John Hiscott

Anne Roulston

Mario D'Addario

Judith Lacoste

Lucie Cohen

\section{ADRESSES}

J. Hiscott : docteur ès sciences. A. Roulston $B$. Sc. . M. D'Addario : B. Sc. J. Lacoste $B$. Sc. L. Cohen : $M$. Sc. Laboratoire d'oncologie moléculaire, institut Lady Davis de recherches médicales et le département de microbiologie et immunologie, université McGill, 3755 chemin Côte. SainteCatherine, Montréal, Québec H3T 1E2,

\title{
La régulation de l'expression de VIH-1 et l'activation des gènes de cytokines
}

Le virus du SIDA VIH-1 pourrait rester un temps indéterminé sous une forme provirale latente, intégrée dans le génome de la cellule infectée mais pratiquement inactive. Sous l'action d'infections intercurrentes, de stimuli divers et de facteurs encore mal connus, la transcription du provirus serait cependant activée, conduisant à l'expression de la pathogénicité. Les facteurs transcriptionnels de la famille NF- $\kappa \mathrm{B} / \mathrm{c}-\mathrm{Rel}$ jouent probablement un grand rôle dans cette activation, de même que dans le contrôle de la transcription de gènes de cytokines qui modulent elles-mêmes, dans un sens positif ou négatif, l'activation de $\mathrm{NF}-\kappa \mathrm{B}$ et du génome proviral. Tous ces processus sont spécifiques de cellules et de tissus par le jeu de l'intervention d'une combinatoire de facteurs de transcription et de plusieurs membres de la famille NF$\kappa B$. Un débrouillage de ce système complexe d'interactions pourrait, peut-être, permettre une intervention thérapeutique destinée à éviter le passage de la phase latente à la phase productive de l'infection par VIH.

e virus d'immunodéficience humaine (VIH) est l'agent responsable du syndrome d'immunodéficience acquise (SIDA) [1, 2]. Ce rétrovirus humain est le rétrovirus le plus complexe jamais découvert; outre les gènes de structure codant pour les protéines internes du virus, les enzymes et les glycoprotéines de l'enveloppe, le VIH contient des séquences codantes pour au moins sept protéines de contrôle - Tat, Rev, Nef,
Vif, Vpr, Vpu, Tev - qui jouent des rôles stratégiques dans la multiplication virale [3].

\section{Pathogénicité du VIH}

La pathogénie du SIDA est due, dans un premier temps, à une destruction sélective, par le virus, de la population de lymphocytes $\mathrm{T}$ qui présente l'antigène de surface CD4 que le VIH utilise comme récepteur. La nature de cette interaction avec le 
récepteur $\mathrm{CD} 4$ a été décrite récemment par Sekaly et al. dans médecine/sciences [4]. L'élimination de toute une population de cellules lymphoïdes jouant un rôle clé dans la réponse immunitaire prédispose la personne atteinte du SIDA à des infections opportunistes débilitantes et finalement mortelles. La lignée des monocytes/macrophages $\mathrm{CD}^{+}{ }^{+}$est aussi très susceptible à l'infection virale, mais, contrairement aux lymphocytes $\mathrm{T}$ infectés, ces cellules ne montrent pas de cytopathologie importante après l'infection ; au contraire, ces populations cellulaires portent le virus continuellement, jouant ainsi un rôle de réservoir qui permet au virus d'être plus persistant et à l'infection de se propager aux tissus périphériques, notamment les poumons, la peau et le cerveau [5]. Dans les lymphocytes $\mathrm{T}$ et les monocytes/macrophages infectés, l'ADN proviral du VIH est intégré dans les chromosomes où il peut rester inactif pendant de longues périodes pour être réactivé par divers stimuli antigéniques comme ceux que représentent d'autres virus [3].

La coexistence stable du provirus VIH et du génome humain nous suggère qu'il peut être impossible de purger complètement l'organisme humain du virus; par conséquent, les patients déjà atteints par le VIH-1 ne pourront pas s'attendre à en être guéris dans le sens conventionnel du mot. Ainsi nous faudra-t-il créer un ensemble de thérapies spécifiques visant à limiter la propagation du virus, et à le maintenir latent. Plusieurs laboratoires se penchent sur la mise au point d'un vaccin qui empêcherait l'infection par VIH, et de substances antivirales chimiothérapeutiques dont le rôle serait d'entraver certaines étapes critiques du cycle de reproduction du virus. Plusieurs revues publiées par médecine/sciences relatent l'infection par le VIH et différentes approches de traitements antiviraux. Les lecteurs peuvent se référer à ces articles pour plus de détails à ce sujet [6-8].

\section{Contrôle de la transcription du VIH}

Dès que le provirus VIH est intégré, l'expression génique, la réplication et $\mathrm{m} / \mathrm{s} n^{\circ} 4$, vol. 8, auril 92 le phénomène de latence sont contrôlés par des interactions entre les protéines virales, des protéines cellulaires, l'effet en cis des séquences d'ADN des éléments répétés ou LTR (long terminal repeat) et la stimulation antigénique des cellules infectées [3]. Le LTR contient plusieurs domaines de régulation servant de sites de fixation pour des protéines cellulaires. Parmi celles-ci, une des plus importantes est NF- $k \mathrm{~B}$ qui reconnaît la séquence GGGACTTTCC présente deux fois dans l'élément activateur (enhancer) du LTR du VIH [9].

Une interaction entre la protéine de trans-activation Tat et les séquences d'ARN localisées directement en amont du site d'initiation de l'ARNm est aussi essentielle à l'expression correcte du LTR du VIH. L'expression fonctionnelle de Tat entraîne une augmentation importante de 100 à 1000 fois de l'expression contrôlée par le LTR, alors que des virus mutants, n'exprimant pas de protéine fonctionnelle, sont incapables de se répliquer [10, 11]. La séquence de réponse à la transactivation TAR (trans-activation response) est présente dans tous les ARNm du VIH-1 sous la forme d'une structure en boucle, stable. La transactivation par Tat requiert d'abord la séquence primaire de cette boucle (nucléotides 31-34), un renflement de trois nucléotides (nucléotides 23-25) et une région intacte de la branche normale. Le renflement joue un rôle important dans la liaison de Tat à TAR mais cette liaison ne suffit pas à engager la transactivation, puisque des mutations dans la boucle ou la branche inhibitent la transactivation par Tat $\left([10,11]\right.$ et $\mathrm{m} / \mathrm{s} n^{\circ} 10$, vol. 5 , p. 779).

\section{L'impact de l'infection par le VIH sur l'expression des gènes de cytokines}

L'infection des cellules myéloïdes par le VIH affecte certaines fonctions fondamentales du système immunitaire, telles que la présentation d'antigène, le chimiotaxisme, et l'élimination des cellules [12]. De plus, l'infection par le VIH-1 des cellules mononucléaires du système sanguin périphérique et des monocytes/macrophages peut avoir des conséquences sérieuses sur la production des cytokines. Les cytokines sont des hormones polypeptidiques produites par les lymphocytes et monocytes/macrophages lors de la réponse à l'infection par un virus ou par d'autres agents pathogènes. La plupart des cytokines exercent plusieurs effets biologiques sur les cellules hématopoiétiques ou endothéliales, et jouent un rôle essentiel dans la production d'une réponse immunitaire efficace, de la stimulation des cellules différenciées et de la prolifération cellulaire. Certaines cytokines synthétisées par les monocytes, tels l'interleukine 1 (IL-1), l'interleukine 6 (IL-6), l'interféron (IFN) et le facteur de nécrose tumorale (TNF) sont des médiateurs importants de la réponse inflammatoire ou antivirale [13]. La production locale de ces cytokines lors de l'infection virale limite l'étape initiale de multiplication du virus et stimule plusieurs éléments cellulaires du système immunitaire acquis.

D'une façon générale, l'infection par le VIH n'affecte ni l'expression ni la sécrétion des cytokines [14], ce qui indique que le VIH est un mauvais inducteur des gènes des cytokines. Cela peut être attribuable à la lenteur de la transmission du VIH comparée à celle de virus plus lytiques. Toutefois, lors d'une stimulation par d'autres virus, par des liposaccharides, des cytokines ou des ARN synthétiques (poly I:C), l'induction des cytokines dans les cellules infectées par le VIH est modifiée. Ainsi, dans les monocytes des patients atteints du SIDA ou de l'ARC (aidsrelated complex, ensemble de symptômes précédant l'apparition d'un SIDA avéré), une corrélation directe a pu être établie entre la progression de la maladie et une diminution progressive de l'inductibilité de la transcription du gène IFN $\alpha$ après infection par le virus de la stomatite vésiculaire. De plus, lorsque des cultures primaires de monocytes infectés par le VIH et traitées par le CSF (colony stimulating factor, spécifique des monocytes), sont traitées avec le poly I:C, on observe un arrêt complet de la transcription de l'IFN $\alpha$, mais non de l'IFN $\beta$ ni de l'IFN $\omega$ [15]. Ces résultats contrastent avec ceux obtenus dans des cellules promonocytaires infectées par le VIH pour lesquel- 


\section{RÉFÉRENCES}

1. Barré-Sinousi F, Cherman JC, Rey F et al. Isolation of a T-lymphotropic retrovirus from a patient at risk for acquired immune deficiency syndrome (AIDS). Science 1983 ; 220 : 868-71.

2. Gallo RC, Salahuddin SZ, Popovic M et al. Frequent detection and isolation of cytopathic retroviruses (HTLV-III) from patients with AIDS and at risk for AIDS Science 1984 ; 224 : 500-3.

3. Vaishnav Y, Wong-Staal F. Biochemistry of AIDS. Ann Rev Biochem 1991; 60 : 577-630.

4. Fleury S, Zerbib A, Lamarre D, Meloche S, Sckaly RP. La moleculc CD4 : un réceptcur aux multiples interactions. médecine/sciences $1992 ; 8: 26-33$.

5. Fauci AS. The human immunodeficiency virus : infectivity and mechanisms of pathogenesis. Science 1988 ; 239 : 617-22.

6. Girard M. Vaccins contre le SIDA : cspoirs et réalités. médecine/sciences 1989 ; 5 152-8.

7. Darlix JL. Nouvelles perspectives en chimiothérapic anti-rétroviralc. médecine/sciences 1989 ; 5 : 213-9.

8. Lévy JP. Traitements du SIDA : recherche de nouveaux médicaments et élaboration de thérapies géniques. médecine/sciences 1991 ; $7: 830-41$.

9. Nabel G, Baltimore D. An inducible transcription factor activates expression of human immunodefiency virus in $\mathrm{T}$ cells. Nature 1987 ; 326 : 711-3.

10. Rosen C, Sodrowski JG, Hascltine WA. Location of cis acting regulating sequences in the human $\mathrm{T}$-cell lymphotropic virus type III long terminal repeat. Cell 1985 ; 41: 813-23.

11. Roy S, Delling U, Chen CH, Rosen CA, Sonenberg N. A bulge structure in HIV-1 TAR RNA is required for Tat binding and Tat-mediated trans-activation. Genes Dev $1990 ; 4: 1365-73$.

12. Meltzer M, Skilman DR, Gomatos PJ, Kalter DC, Gendelman HE. Role of mononuclear phagocytes in the pathogenesis of the human immunodefiency virus infection. Ann Rev Immunol 1990 ; 8 : 169-97.

13. Arai KI, Lec F, Miyajima A, Miyatake $\mathrm{S}$, Arai N, Yokota T. Cytokines : coordinators of immune and infammatory responscs. Ann Rev Biochem 1990 ; 59 : 783-850. 14. D'Addario M, Roulston A, Wainber MA, Hiscott J. Coordinate enhancement of cytokine gene expression in human immunodeficiency virus type 1 -infected promonocytic cclls. I Virol 1990; 64: 6080-9.

15. Gendelman H, Fricdman RM, Joc S, et al. A selective defect in interferon $\alpha$ production in human immunodeficiency virus infected monocytes. $J$ Exp Med $1990 ; 172$ : 1433-42.

16. Osborn LS, Kunkel S, Nabel GJ. Tumor necrosis factor $\alpha$ and interleukin 1 stimulate the human immunodeficiency virus enhancer by activation of the nuclear factor кB. Proc Natl Acad Sci USA 1989; 86 : 2336-40. les l'infection par le virus de Sendai ou un traitement aux LPS conduisent à une augmentation coordonnée de la transcription de l'IFN $\beta$, du TNF, de l'IL-6 et de l'IL-1 $\beta$ [14].

La production et la sécrétion de cytokines par les cellules infectées pourraient aussi influencer la pathogénicité du VIH in vitro. En effet, le traitement de cellules par le TNF- $\alpha$, l'IL-1 ou le GM-CSF (granulocyte/macrophage colony stimulating factor) augmente la transcription et la réplication du VIH $[16,17]$. Cependant, l'addition de TGF- $\beta$ (transforming growth factor), de TNF- $\alpha$ et d'IFN- $\gamma$ à des cultures de monocytes avant leur infection par le VIH, inhibe la multiplication du virus [18]. De même, la présence d'IFN $\alpha 2$ dans le milieu de culture ralentit la progression de l'infection dans les promonocytes et a un effet synergique avec l'azidothymidine sur la réplication du virus [19]. L'ensemble de ces résultats indique que des molécules immunorégulatrices solubles peuvent avoir des effets opposés sur la réplication virale et, ainsi, influencer la pathogénicité du virus, qui évoluerait alors d'un cycle latent vers un cycle lytique.

\section{Biochimie et biologie moléculaire de NF-kB/rel}

Les cytokines, les esters de phorbol, une infection virale et d'autres agents mitogènes peuvent induire la réplication du VIH - notamment par l'activation du complexe NF- $\kappa$ B qui se fixe et active la séquence activatrice du VIH. La famille des facteurs de transcription $\mathrm{NF}-\kappa \mathrm{B} / \mathrm{rel}$ interagit avec une séquence d'ADN de 10

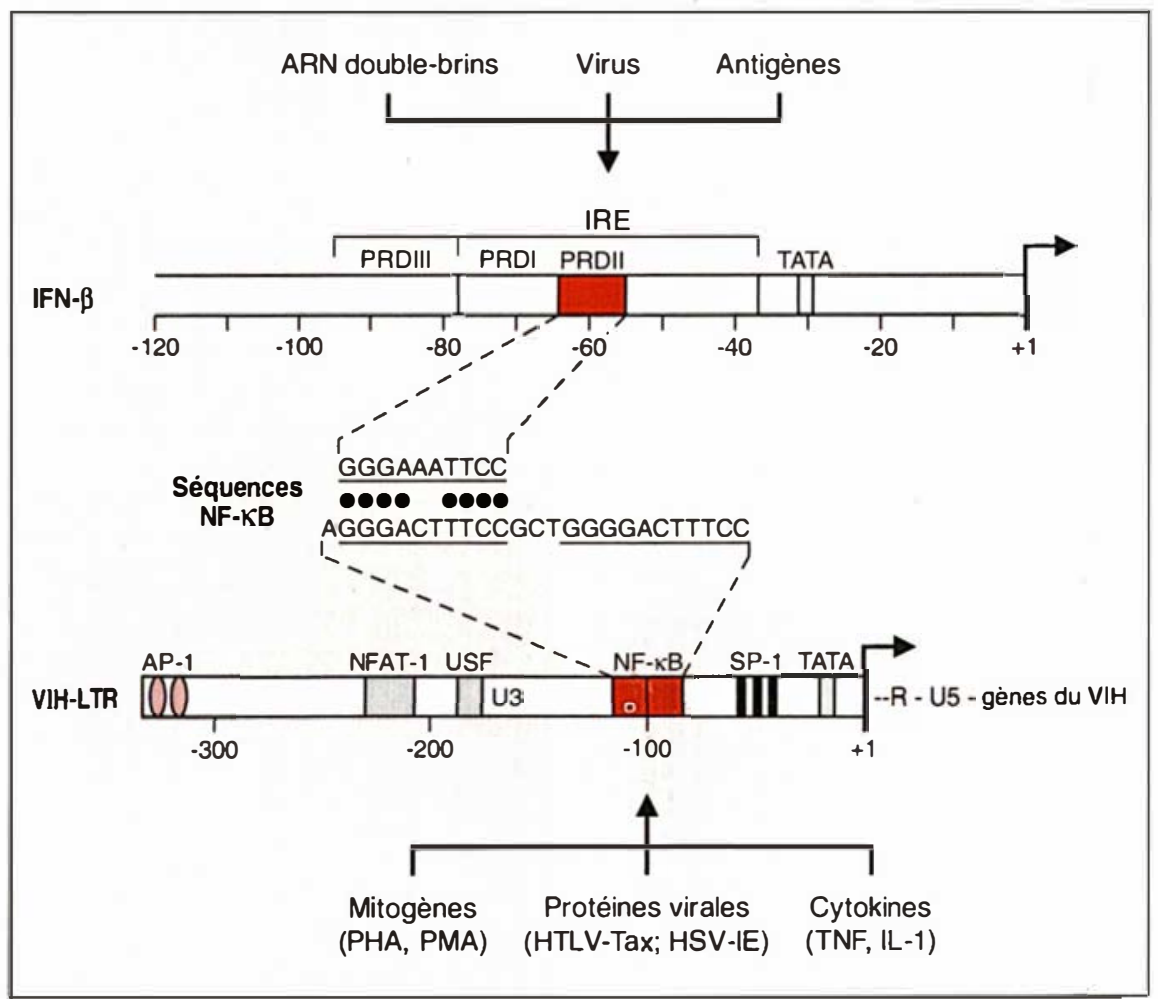

Figure 1. Représentation schématique des régions promotrices des gènes de I'interféron $\beta$ et du VIH-1. L'activation transcriptionnelle est contrôlée par plusieurs facteurs qui interagissent avec les séquences activatrices des promoteurs de I'IFN $\beta$ et du VIH. L'un de ces facteurs, NF- $\alpha B$, induit par les ARN double brins, les virus, et une variété $d^{\prime}$ autres antigènes, se fixe à une séquence (consensus GGGANNYYCC) contenue dans le domaine PRDIl de IFN $\beta$ pour stimuler la transcription (L. Cohen et J. Hiscott, soumis pour publication). NF-kB se fixe aussi à deux sites présents dans le LTR du VIH qui augmentent la réplication virale [3]. (U3, $R$ et U5 : régions des LTR des rétrovirus; IRE : interferonresponse element ; PRD : proximal regulatory domain ; AP1, NFAT, USF, SP1 sont des protéines ou des complexes protéiques contrôlant la transcription). 
nucléotides (consensus 5'-GGGRNN YYCC-3'), et participe à l'activation de plusieurs gènes codant pour des protéines impliquées dans les réactions inflammatoires et les fonctions régulatrices, dont les protéines de la phase aiguë, les cytokines immunomodulatrices comme les IFN- $\beta$, IL-2, IL-6, IL-8, TNF- $\alpha$ et GM-CSF, les récepteurs de surface impliqués dans la transduction de signaux et la reconnaissance immunitaire (antigènes du complexe majeur d'histocompatibilité, récepteurs de l'IL-2, récepteurs du TNF) ; NF- $\kappa$ B/rel participe aussi à l'activation de domaines enhancer de plusieurs virus (VIH, SV40 et cytomégalovirus) [20]. La figure 1 représente les séquences et la position des éléments régulateurs NF$\kappa \mathrm{B}$ présents dans les promoteurs du gène de l'interféron $\beta$ et du LTR du VIH.

L'induction de NF- $\kappa$ B semble essentiellement se produire au niveau posttraductionnel ( $\mathrm{m} / \mathrm{s} n^{\circ} 1$, vol. 7, p. 67). La figure 2 illustre la biochimie de l'induction de NF- $\kappa$ B et son interaction avec l'ADN. Dans le cytoplasme de la majorité des cellules, on retrouve un complexe de trois sousunités de NF- $\kappa \mathrm{B}$ : deux protéines qui se lient à l'ADN, une de 48 à $55 \mathrm{kDa}$ (p50), une de 65 à $68 \mathrm{kDa}$ (p65) et une autre, appelée $\mathrm{I} \kappa \mathrm{B}$, qui interagit spécifiquement avec p65. I $\mathrm{B}$ semble être responsable de la localisation du complexe NF- $\kappa$ B inactif dans le cytoplasme ; la phosphorylation de $\mathrm{I} \kappa \mathrm{B}$ produit une dissociation de I $k$ B du couple p50-p65 et une translocation nucléaire rapide de NF$\kappa \mathrm{B}$. Le clonage moléculaire des composantes $\mathrm{p} 50$ et p65 de $\mathrm{NF}-\kappa \mathrm{B}$ a montré que l'extrémité $\mathrm{N}$-terminale de ces protéines avait une forte homologie de séquence avec le protooncogène c-rel (p85) [21].

L'obtention des séquences codantes des sous-unités de NF- $\mathrm{B}$ a, par ailleurs, permis de démontrer que la transcription des gènes codant pour ces protéines était aussi soumise à d'importantes régulations ; la transcription des gènes codant pour les sous-unités p50 et $\mathrm{I} \kappa \mathrm{B}$ de $\mathrm{NF}-\kappa \mathrm{B}$ et pour c-rel est induite par les esters de phorbol et par d'autres mitogènes [21-24]. De plus, deux formes distinctes de la sous-unité p50 sont produites à partir d'une protéine pré-

$\mathrm{m} / \mathrm{s} n^{\circ} 4$, vol. 8, auril 92



Figure 2. Schéma illustrant l'induction du facteur de transcription NF$\kappa B$. A l'état latent, le facteur de transcription NF- $\kappa B$ est constitué d'un hétérotrimère cytoplasmique contenant les sous-unités $p 50$ et p65 ainsi qu'un inhi-

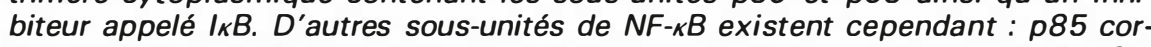
respond au produit de l'oncogène cellulaire c-rel. $p 72$ serait analogue à $p 65$, et les sous-unités p56 et $p 47$ seraient des dérivés de $p 50$. Des signaux induits par des cytokines, virus ou substances mitogènes, au niveau de la membrane cellulaire, activent la phosphorylation de l'inhibiteur $I_{\kappa} B$, ce qui provoque sa dissociation du complexe NF- $\kappa B$ et la migration de ce dernier vers le noyau cellulaire. Dans le noyau, I'hétérodimère formé des sous-unités $p 50$ et p65 se fixe à une séquence spécifique $d^{\prime} A D N$. Cette fixation à l'ADN en amont $d u$ gène cible permet au complexe NF-kB d'interagir avec la machinerie de transcription de la cellule afin de produire l'ARN messager qui servira à la synthèse protéique. La transcription se termine lorsque l'hétérodimère se dissocie de I'ADN. Trois mécanismes de dissociation sont possibles : dissociation induite par l'inhibiteur $I_{k} B$, par un homodimère de la sous-unité $p 50$, ou par la sousunité p85 (c-Rel).

curseur de $100 \mathrm{kDa}$ dont le gène a été isolé récemment ([24] et $\mathrm{m} / \mathrm{s} n^{\circ} 1$, vol. 7, p. 67). Les deux formes de p50 résultent d'une double régulation du gène, l'une au niveau posttranscriptionnel agissant par un processus d'épissage différentiel du transcrit primaire et l'autre au niveau post-transcriptionnel, par la maturation des précurseurs.

Considérant le nombre de stimuli capables d'induire $\mathrm{NF}-\kappa \mathrm{B}$, il est très probable qu'il existe plusieurs voies d'activation du complexe protéique en fonction du type cellulaire. Récemment, des études ont montré que certains agents - tels que le peroxyde d'hydrogène, qui accroît la concentration de radicaux oxygène dans la cellule - peuvent aussi induire l'activité de fixation à l'ADN de façon importante [25]. L'augmentation temporaire de la concentration en radicaux oxygène résultant d'une gamme de stimuli physiologiques pourrait, éventuellement, représenter le carrefour des diverses voies utilisées par ces différents agents pour relayer l'activation de $\mathrm{NF}-\kappa \mathrm{B}$.

L'induction de l'activité de fixation de NF- $\kappa$ B à l'ADN par les esters de phorbol et le TNF varie selon le type cellulaire; ces inducteurs peuvent aussi stimuler de façon importante la production du VIH in vitro [26]. Ces différences d'inductibilité et de spécificité cellulaire découlent sûrement d'une hétérogénéité dans la famille des facteurs de transcription NF- $\mathrm{NB}$. Plusieurs sous-unités de $\mathrm{NF}-\kappa \mathrm{B}$ qui se lient à l'ADN ont été purifiées par des méthodes biochimiques et molé- 


\section{RÉFÉRENCES}

17. Poli G, Kinter A, Justement JS, el al. Tumor necrosis factor $\alpha$ functions in an autocrine manner in the induction of human immunodeficiency virus expression. Proc Natl Acad Sci USA 1990 ; 87 : 782-5.

18. Wong GHW, Krowka JF, Stites DP Gocddel DV. In vitro anti-HIV activitics of TNF- $\alpha$ and IFN- $\boldsymbol{\gamma}$. J Immunol $1988 ; 140$ 120-4.

19. Dubreuil M, Sportza L, D'Addario M el al. Inhibition of HIV-1 transmission by interferon and 3'-azido-3'-deoxythimidine during de novo infection of promonocytic cclls. Virology 1990 ; 179 : 388-94.

20. Bacucrle PA. The inducible transcription activator NF- $\kappa \mathrm{B}$ : regulation by distinct protcin subunits. Biochim Biophys Acta 1991 1072: 63-90.

21. Kieran M, Blank V, Logeat F, el al. The DNA binding subunit of NF- $\kappa \mathrm{B}$ is identical to factor KBF1 and homologous to the rel oncogenc product. Cell 1990; 62 : 1007-18.

22. Inouc JI, Kerr LD, Ransone LJ, Bengal E, Hunter T, Verma IM. c-rel activates but $v$-rel suppresses transcription from $\kappa \mathrm{B}$ sitcs. Proc Natl Acad Sci USA 1991; 88 : 3715-9.

23. Haskill S, Beg A, Tompkins $\mathrm{H}$, el al. Characterization of an immediate-carly gene induced in adherent monocytes that encodes IkB-like activity. Cell 1991; 65 1281-90.

24. Schmid RM, Perkins ND, Duckett CS, Andrews PC, Nabel GJ. Cloning of an NF$\kappa \mathrm{B}$ subunit which stimulates HIV transcription in synergy with p65. Nature $1991 ; 352$ : 733-6.

25. Schreck R, Picber P, Bacucrle P. Reactive oxygen intermediates as apparently widely used messengers in the activation of the NF- $\kappa \mathrm{B}$ transcription factor and HIV-1. EMBO J 1991; 10 : 2247-58

26. Lacostc J, D'Addario M, Roulston A Wainberg MA, Hiscott J. Cell-specific differences in activation of $\mathrm{NF}-\kappa \mathrm{B}$ regulatory clements of human immunodeficiency virus and beta interferon promoters by tumor necrosis factor. I Virol 1990 ; 64 : 4726-34 27. Cohen L, Lacoste J, Parniak M, Daigneault L, Skup D, Hiscott J. Stimulation of interferon $\beta$ transcription in vitro by purified NF- $\mathrm{NB}$ and a novel $\mathrm{TH}$ protein. Cell Growth Differ 1991; 2 : 323-33.

28. Roulston A, D'Addario M, Boulcrice F, Caplan S, Wainberg MA, Hiscott J. Induc tion of monocytic differentiation and NF- $\kappa \mathrm{B}$ like activity by HIV-1 infection of myclomonoblastic cells. J Exp Med 1992 (sous presse).

29. Leblanc JF, Cohen L, Rodrigues M, Hiscott J. Synergism between distinct cnhanson domains in viral induction of the human interferon $\beta$ genc. Mol Cell Bio $1990 ; 10: 3987-93$

30. Schmitz ML, Bacuerle P. The p65 subunit is responsible for the strong transcription activating potential of $\mathrm{NF}-\kappa \mathrm{B}$ culaires, et leurs différentes affinités pour l'ADN ont été mises en évidence [27].

La modulation de NF- $\kappa$ B dans les cellules infectées par le VIH-1 en relation avec l'expression de cytokines ou de la différenciation des monocytes fait encore l'objet de recherches. Pour comprendre les rclations entre la transcription des gènes, l'induction de $\mathrm{NF}-\kappa \mathrm{B}$ et l'infection par le VIH-1 de ccllules myéloïdes, l'induction de l'expression des gènes de cytokines par un ester de phorbol (PMA) et le TNF- $\alpha$ a été étudiée en utilisant un nouveau modèle d'infection des myélomonoblastes par le VIH-1 [28]. Les PBL-985 (peripheral blood leukocytes-985) constituent une population de cellules immatures qui sont capables de se différencier en monocytes ou en granulocytes. En vertu de l'expression de CD4 à leur surface, ces cellules ont été infectées par la souche III-B du VIH-1, créant ainsi une lignée infectée chroniquement (PBL-IIIB). Il a été établi d'après leur morphologie, leur coloration, l'expression de marqueurs de surface spécifiques des monocytes et la transcription du proto-oncogène $c$ fms (qui code pour le récepteur de CSF-1, facteur de croissance spécifique des monocytes), que les PLBIIIB avaient un phénotype plus monocytique que leurs parents myéloblastes. De plus, ces cellules ne réagissent plus aux promoteurs de la différenciation granulocytaire. Dans les cellules infectées par le VIH-1, de nouveaux complexes protéines-ADN ont été identifiés en utilisant lc site de fixation pour NF- $\kappa$ B présent dans le gène de l'interféron $\beta$. En particulier, l'expression constitutive d'un nouveau complexe NF- $\kappa$ B (p90-p100) a été découverte; cc complexe a une plus grande affinité pour le site PRDII NF- $\kappa$ B du promoteur IFN- $\beta$ que pour la séquence enhancer du VIH (figure 1). Le traitement au TNF et au PMA des cellules myéloïdes infectées par le VIH modifie le taux de transcription des gènes de cytokines spécifiques de la lignée myéloïde, et parfois aussi la sécrétion de ces dernières. De façon surprenante, la transcription du gène de l'IL- $1 \beta$ est fortement stimulée par ces deux inducteurs dans les cellules infectées par le VIH, alors que la plus grande partie de l'IL-1 $\beta$ synthétisée demeure dans les cellules. Ces études nous poussent à croire que l'infection de monoblastes par le VIH-1 pourrait influencer ces cellules à évoluer vers un phénotype monocytique plus mûr et que certaines combinaisons des sous-unités du complexe NF- $\mathrm{NB}$ pourraient contribuer à une expression génique différentielle [28].

Par quels moyens NF- $\kappa$ B arrive-t-il à conserver une sélectivité dans l'activation de la transcription de gènes cibles ? La spécificité découle en partie de l'interaction coopérative de $N F-\kappa B$ avec d'autres facteurs de transcription qui se fixent à des séquences adjacentes au site $\mathrm{NF}-\kappa \mathrm{B}$ en cis de gènes inductibles, comme cela a été montré pour l'interféron $\beta$ [29]. Dans le cas de l'IFN $\beta$, l'activation du promoteur nécessite un synergisme entre $\mathrm{NF}-\kappa \mathrm{B}$ et le facteur de régulation de l'interféron (IRF-1) La spécificité de transcription et de liaison à l'ADN pourrait aussi découler des interactions entre les différentes sous-unités NF- $\kappa$ B. L'étude de l'interaction de polypcptides de 45 à $85 \mathrm{kDa}$ (p47, p50, p65, p72 et p85) avec le site NF- $\kappa$ B a permis de montrer que : (1) ces molécules ont des affinités différentes pour l'ADN et (2) forment de multiples homo- ou hétérodimères qui se fixent eux aussi à l'ADN. De plus, ces polypeptides purifiés peuvent stimuler la transcription in vitro sous forme d'hétérodimères mais non d'homodimères (L. Cohen et J. Hiscott, soumis pour publication). L'analyse de mutants a récemment démontré que la portion carboxy-terminale de la sous-unité p65 de NF- $\kappa$ B est le domaine de transactivation de la protéine [30]. L'étude de ces différentes formes du facteur permettra sans doute de comprendre le rôle de $\mathrm{NF}_{-\kappa} \mathrm{B}$ dans la latence ou la réactivation du provirus VIH. De telles recherches pourraient découler des méthodes thérapeutiques permettant de faire perdurer la latence du VIH et, par conséquent, de bloquer sa pathogénicité

\section{TIRÉS A PART}

J. Hiscott. 


\section{Summary}

Regulation of HIV -1 expression and cytokine gene activation by $N F-\kappa B / r e l$ transcription factors

Control of HIV provirus expression is regulated by the complex interplay between HIV regulatory proteins, cellular proteins acting on DNA sequences within the viral long terminal repeat (LTR) and antigenic stimulation of HIV infected cells. The LTR contains multiple regulatory domains that serve as binding sites for cellular proteins. One of the most important of these cellular proteins is NF- $k \mathrm{~B}$ which recognizes the sequence GGGACTTTCC present twice within the HIV enhancer element. NF- $\kappa \mathrm{B}$ represents a family of DNA binding proteins that are related to the rel protooncogene ; the proteins participate in the activation of numerous genes involved in inflammatory reactions and immune regulatory functions including the acute phase proteins, immunomodulatory cytokines and cell surface receptors involved in signal transduction and immune recognition. Induction of NF- $\kappa$ B DNA binding activity occurs in response to virus infection, cytokine treatment, phorbol esters and other antigenic stimuli that increase HIV replication. NF- $\kappa \mathrm{B}$ activation has been characterized largely as a posttranslational event. A complex of three NF- $\kappa B$ subunits exists in the cytoplasm of most cells : two DNA binding proteins, p50 and $\mathrm{p} 65$, and a non-DNA binding regulatory subunit termed I $\alpha$ B that interacts specifically with $\mathrm{p} 65$. I $\kappa \mathrm{B}$ is phosphorylated as a result of signal transduction leading to dissociation of the p50p65 heterodimer and rapid nuclear localization of the DNA binding complex. Purification and cloning experiments have revealed several additional $\mathrm{NF}-\mathrm{\kappa} \mathrm{B}$ related proteins and multiple interactions that regulate HIV LTR mediated transcription and cytokine gene activation. 